\title{
FORMATION CONTROL OF MULTIPLE UNICYCLE-TYPE ROBOTS USING LIE GROUP
}

\author{
Youwei DonG*, Ahmed Rahmani \\ Ecole Centrale de Lille, Cité Scientifique - CS20048, 59651 Villeneuve d'Ascq Cedex, France \\ * corresponding author: youwei.dong@ec-lille.fr
}

ABSTRACT. In this paper the formation control of a multi-robots system is investigated. The proposed control law, based on Lie group theory, is applied to control the formation of a group of unicycle-type robots. The communication topology is supposed to be a rooted directed acyclic graph and fixed. Some numerical simulations using Matlab are made to validate our results.

KEYWORDS: formation control; multi-robot system; unicycle-type robot; Lie group; Lie algebra.

\section{INTRODUCTION}

The various ways to control and coordinate a group of mobile robots widely have been studied in recent years. This has brought a breadth of innovation, providing considerable attention for the potential applications, such as flocking systems control, surveillance, search and rescue, cooperative construction, distributed sensor fusion, etc. When comparing the mission outcome of a multi-robot system (MRS) to that of a single robot, it is clear that cooperation among multiple robots can perform complex tasks that it would otherwise be impossible for a single powerful robot to accomplish. The fundamental idea behind multirobotics is to allow the individuals to interact with each other to find solutions of complex problems. Each of them senses the relative positions of his neighbors, and achieves the desired formation by controlling the relative positions [1 3]. In formation control, various control topologies can be adopted, depending on the specific environment and tasks. Theoretical views of MRS behavior are divided into centralized and decentralized systems. In a centralized system, a powerful core unit makes decisions and communicates with the others. In the decentralized approach, the robots can communicate and share information with each other [4]. We will focus on distributed system control due to its advantages, such as feasibility, accuracy, robustness, cost, and so on.

Many studies have been devoted to the control and coordination of multi-agent systems and multi-robot systems (e.g. 1, 44 9]). Some of these results have been used to control vehicles (holonomic, nonholonomic mobile robots, etc.). In this paper, our goal is to control a group of unicycle robots to achieve a desired formation. Motivated by references [10 14], we focus on a rigid body with kinematics evolving on Lie groups. This is based on regarding the set of rigid body posture as the Lie group $S E(2)$, which leads to a set of kinematic equations. These equations are expressed in terms of standard coordinated invariant linear operators on the Lie algebra $s e(2)$. This approach allows a global description of rigid body motion which does not suffer from singularities. It provides a geometric description of rigid motion which greatly simplifies an analysis of the mechanisms [10]. Paper [1] proposed an elegant control law based on Lie algebra theory for consensus of a multi-agent system. It has the holonomic constraints, while nonholonomic constraints are not considered. In [12, Lie algebra is used to study the path following control of one mobile robot. In [15], distributed formation control of multi-nonholonomic robots is studied. However the the control law is a leader-follower approach, and the multi-leader case is not considered. In this paper, a Lie group method is used to control multiple unicycletype robots. The communication topology is defined as a rooted directed acyclic graph (DAG). Due to the nonholonomic property of this type of robot, a new local control law is proposed to make the nonlinear system converge to the desired formation.

The outline of this paper is as follows. In Section 2 some preliminary results are summarized and the formation control problem for a group of unicycle-type robots is stated. In Section 3, a formation control strategy is proposed and the stability is analyzed. The simulation and results are given in Section 4 Concluding remarks are finally provided in Section 5 .

\section{Preliminary AND PROBlem STATEMENT}

2.1. LiE GROUPS

Definition 1 [10]. A manifold of dimension of $n$ is a set $M$, which is locally homeomorphic to $\mathbb{R}^{n}$. A Lie group is a group $G$ which is also a smooth manifold and for which the group operations $(g, h) \mapsto g h$ and $g \mapsto g^{-1}$ are smooth. Left action of $G$ on itself $L_{g}$ : $G \rightarrow G$ is defined by $L_{g}(h)=g h$, and right action is defined the same way. Adjoint action $\operatorname{Ad}_{g}: G \rightarrow G$ is $\operatorname{Ad}_{g}(h)=g h g^{-1}$.

Here are two examples, the special orthogonal group $S O(n)=\left\{R \in G L(n, \mathbb{R}): R R^{T}=I, \operatorname{det} R=+1\right\}$ and the special Euclidean group $S E(n)=\{(p, R): p \in$ $\left.\mathbb{R}^{n}, R \in S O(n)\right\}=\mathbb{R}^{n} \times S O(n)$. 


\subsection{Lie ALGEBRA ASSOCIATED WITH A LIE GROUP}

A Lie algebra $\mathfrak{g}$ over $\mathbb{R}$ is a real vector space $\mathfrak{g}$ together with a bilinear operator [,]: $\mathfrak{g} \times \mathfrak{g}$ (called the bracket) such that for all $x, y, z \in \mathfrak{g}$, we have:

- Anti-commutativity: $[x, y]=-[y, x]$;

- Jacobi identity: $[[x, y], z]+[[y, z], x]+[[z, x], y]=0$.

A Lie algebra $\mathfrak{g}$ is said to be commutative (or abelian) if $[x, y]=0$ for all $x, y \in \mathfrak{g}$. We can define $\operatorname{ad}_{A} B=[A, B]=A B-B A$ where $A, B \in \mathfrak{g l}(n, \mathbb{R})$, which is the vector space of all $n \times n$ real matrices, $\mathfrak{g l}(n, \mathbb{R})$ forms a Lie algebra. Clearly, we have $[x, x]=0$. The Lie algebra of $S O(2)$, denoted by $s o(2)$, may be identified with a $2 \times 2$ skew-symmetric matrix of the form $\hat{\omega}=\left[\begin{array}{cc}0 & -\omega \\ \omega & 0\end{array}\right]$ with the bracket structure $\left[\hat{\omega}_{1}, \hat{\omega}_{2}\right]=\hat{\omega}_{1} \hat{\omega}_{2}-\hat{\omega}_{2} \hat{\omega}_{1}$, where $\hat{\omega}_{1}, \hat{\omega}_{2} \in s o(2)$. The Lie algebra of $S E(2)$, denoted by $s e(2)$, can be identified with a $3 \times 3$ matrix of the form $\hat{\xi}=\left[\begin{array}{ll}\hat{\omega} & v \\ 0 & 0\end{array}\right]$, where $\omega \in \mathbb{R}, v \in \mathbb{R}^{2}$, with the bracket $\left[\hat{\xi}_{1}, \hat{\xi}_{2}\right]=\hat{\xi}_{1} \hat{\xi}_{2}-\hat{\xi}_{2} \hat{\xi}_{1}$.

The exponential map: $\exp : T_{e} G \rightarrow G$ is a local diffeomorphism from the neighborhood of zero in $\mathfrak{g}$ onto the neighborhood of $e$ in $G$. The mapping $t \rightarrow$ $\exp (t \hat{\xi})$ is the unique one-parameter subgroup $\mathbb{R} \rightarrow G$ with tangent vector $\hat{\xi}$ at time 0 . For $\hat{\omega} \in s o(2)$ and $\hat{\xi}=(\hat{\omega}, v) \in \operatorname{se}(2)$, we have

$$
\begin{aligned}
\exp \hat{\omega} t & =\left[\begin{array}{cc}
\cos \omega t & -\sin \omega t \\
\sin \omega t & \cos \omega t
\end{array}\right], \\
\exp \hat{\xi} t & =\left[\begin{array}{cc}
\exp \hat{\omega} t & A(\omega) v \\
0 & 1
\end{array}\right],
\end{aligned}
$$

where

$$
A(\omega)=\frac{1}{\omega}\left[\begin{array}{cc}
\sin \omega t & -(1-\cos \omega t) \\
(1-\cos \omega t) & \sin \omega t
\end{array}\right] .
$$

\subsection{GRAPH THEORY}

The communication topology among $N$ robots will be represented by a graph. Let $\mathcal{G}=(\mathcal{V}, \mathcal{E}, \mathcal{A})$ be a graph of order $N$ with the finite nonempty set of nodes $\mathcal{V}(\mathcal{G})=\left\{v_{1}, \ldots, v_{N}\right\}$, the set of edges $\mathcal{E}(\mathcal{G}) \subset \mathcal{V} \times \mathcal{V}$, and an adjacency matrix $\mathcal{A}=\left(a_{i j}\right)_{N \times N}$. If for all $\left(v_{i}, v_{j}\right) \in \mathcal{E},\left(v_{j}, v_{i}\right) \in \mathcal{E}$ as well, the graph is said to be undirected, otherwise it is called directed. Here, each node $v_{i}$ in $\mathcal{V}$ corresponds to a robot- $i$, and each edge $\left(v_{i}, v_{j}\right) \in \mathcal{E}$ in a directed graph corresponds to an information link from robot- $i$ to robot- $j$, which means that robot- $j$ can receive information from robot- $i$. In contrast, the pairs of nodes in an undirected graph are unordered, where an edge $\left(v_{i}, v_{j}\right) \in \mathcal{E}$ denotes that each robot can communicate with the other one. The adjacency matrix $\mathcal{A}$ of a digraph $\mathcal{G}$ is represented as

$$
\mathcal{A}=\left[\begin{array}{cccc}
a_{11} & a_{12} & \cdots & a_{1 n} \\
a_{21} & a_{22} & \cdots & a_{2 n} \\
\vdots & \vdots & \ddots & \vdots \\
a_{n 1} & a_{n 2} & \cdots & a_{n n}
\end{array}\right],
$$

where $a_{i j}$ is the weight of link $\left(v_{i}, v_{j}\right)$ and $a_{i i}=0$ for any $v_{i} \in \mathcal{V}, a_{i j}>0$ if $\left(v_{i}, v_{j}\right) \in \mathcal{E}$ and $a_{i j}=0$ otherwise. $\mathcal{A}$ of a weighted undirected graph is defined by analogy, except that $a_{i j}=a_{j i}, \forall i \neq j[16$. A directed path from node $v_{i}$ to $v_{j}$ is a sequence of edges $\left(v_{i}, v_{j 1}\right),\left(v_{j 1}, v_{j 2}\right), \ldots,\left(v_{j l}, v_{j}\right)$ in a directed graph $\mathcal{G}$ with distinct nodes $v_{j k}, k=1, \ldots, l$. A directed graph is called acyclic if it contains no directed cycle. A rooted graph is a graph in which one vertex is distinguished as the root.

\subsection{Problem Statement}

A unicycle-type mobile robot is composed of two independent actuated wheels on a common axle which is rigidly linked to the robot chassis. In addition, there are one or several passive wheels (for example, a caster, Swedish or spherical wheel) which are not controlled and just serve for sustentation purposes [17]. We study the formation control problem of a group of such robots and each one is equipped with a local controller for deciding the velocities. We consider each robot as a node of a directed graph $\mathcal{G}$, then the communication topology of a group of $N$ robots could be expressed by an adjacency matrix $\mathcal{A}=\left(a_{i j}\right)_{N \times N}$, where $a_{i i}=0$ and $a_{i j}=1$ if $\left(v_{i}, v_{j}\right) \in \mathcal{E}$ or 0 otherwise. The purpose is to design the strategy of the control applied to each robot in order that this group of mobile robots could execute a predefined task of formation control.

\subsection{Kinematic MODEL ON A LiE GROUP}

In order to describe the kinematic properties of the unicycle-type robot, we consider a reference point $O_{R}$ at the mid-distance of the two actuated wheels. Then we define two frames: $\mathcal{F}_{I}=\{O, X, Y\}$ and $\mathcal{F}_{R}=$ $\left\{O_{R}, X_{R}, Y_{R}\right\}$, as shown in Figure 1. $\mathcal{F}_{I}=\{O, X, Y\}$ is an arbitrary inertial basis on the plane as the global reference frame and $\mathcal{F}_{R}=\left\{O_{R}, X_{R}, Y_{R}\right\}$ is a frame attached to the mobile robot with its origin located at $O_{R}$, and the basis $\left\{X_{R}, Y_{R}\right\}$ defines two axes relative to $O_{R}$ on the robot chassis and is thus the robot's local reference frame. The position of the robot in the global reference is specified by coordinates $x_{I}$ and $y_{I}$, and the angular difference between the global and local reference frames is given by $\theta_{I}$. Then the pose of the robot could be described as an element of the Lie group $S E(2)$ :

$$
g=\left[\begin{array}{ll}
R & p \\
0 & 1
\end{array}\right]=\left[\begin{array}{ccc}
\cos \theta_{I} & -\sin \theta_{I} & x_{I} \\
\sin \theta_{I} & \cos \theta_{I} & y_{I} \\
0 & 0 & 1
\end{array}\right],
$$

where $p=\left[x_{I}, y_{I}\right]^{T}$ denotes the position of the robot in the global reference frame, and $R=\left[\begin{array}{cc}\cos \theta_{I} & -\sin \theta_{I} \\ \sin \theta_{I} & \cos \theta_{I}\end{array}\right]$ is the rotation matrix of the frame $\mathcal{F}_{R}$ relative to frame $\mathcal{F}_{I}$. Then the motion of a robot could be described by $g(t)$, which is a curve parameterized by time $t$ in $S E(2)$. 


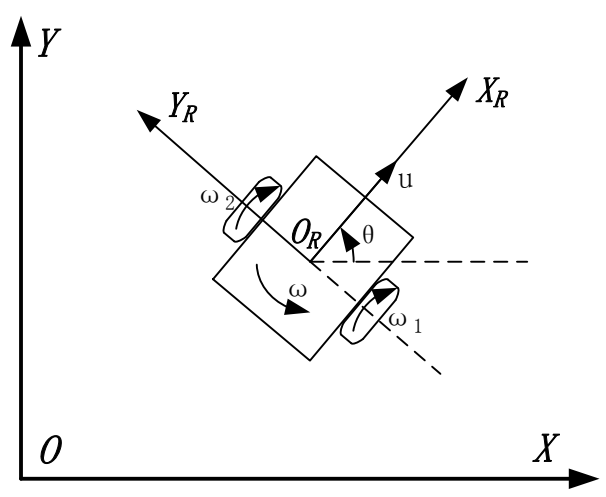

FiguRE 1. Representation of the frames.

Each pure rotational motion of a robot on a plane can be given by a $2 \times 2$ orthogonal matrix $R \in S O(2)$. Let $\omega \in \mathbb{R}$ be the rotation velocity of the robot's chassis and then the exponential map exp : so(2) $\rightarrow$ $S O(2), \hat{\omega} \rightarrow \exp (\hat{\omega} t)$ which is defined by Equation 1 where $\hat{\omega}=\left[\begin{array}{cc}0 & -\omega \\ \omega & 0\end{array}\right] \in s o(2)$ correspond to the robot chassis rotation. This map represents the rotation from the initial $(t=0)$ configuration of the robot to its final configuration with the rotation velocity $\omega$.

The rigid motions consist of rotation and translation. A general motion could also be described by an exponential map exp $: \operatorname{se}(2) \rightarrow S E(2), \hat{\xi} \rightarrow \exp (\hat{\xi} t)$ defined in Equation 22 where $\hat{\xi}=\left[\begin{array}{cc}\hat{\omega} & v \\ 0 & 0\end{array}\right] \in$ se $(2)$ represents the velocities of movement, and $v=-\hat{\omega} p+\dot{p}=$ $\left[\begin{array}{c}\omega y_{I}+\dot{x}_{I} \\ -\omega x_{I}+\dot{y}_{I}\end{array}\right]$ where $\left(x_{I}, y_{I}\right)$ is the position of the robot and $v$ represents the velocity of a (possibly imaginary) point on the rigid body which is moving through the origin of the world frame. $\exp (\hat{\xi} t)$ is a mapping from the initial configuration of the robot to its final configuration. That is, if we suppose that the initial configuration of the robot is $g(0)$, then the final configuration is given by

$$
g(t)=e^{\hat{\xi} t} g(0) .
$$

The kinematic model of the unicycle-type robot is given by

$$
\left\{\begin{array}{l}
\dot{x}_{I}=u \cos \theta_{I} \\
\dot{y}_{I}=u \sin \theta_{I} \\
\dot{\theta}_{I}=\omega
\end{array}\right.
$$

where $u$ characterizes the robot's longitudinal velocity. The variables $u$ and $\omega$ are related to the angular velocity of the actuated wheels via the one-to-one transformation:

$$
\left[\begin{array}{l}
u \\
\omega
\end{array}\right]=\left[\begin{array}{cc}
r_{w} / 2 & r_{w} / 2 \\
r_{w} / L & -r_{w} / L
\end{array}\right]\left[\begin{array}{l}
\omega_{1} \\
\omega_{2}
\end{array}\right]
$$

where $r_{w}$ is the wheels' radius, $L$ is the distance between the two actuated wheels, and $\omega_{1}$ and $\omega_{2}$ are respectively the angular velocity of the right wheel and the left wheel.

We differentiate the matrix given in Equation 3. and obtain the kinematic model of a unicycle-type robot on the Lie group:

$$
\dot{g}(t)=\hat{\xi} g(t)
$$

where $\hat{\xi}$ is the control input matrix given by

$$
\hat{\xi}=\left[\begin{array}{ccc}
0 & -\omega & \omega y_{I}+u \cos \theta_{I} \\
\omega & 0 & -\omega x_{I}+u \sin \theta_{I} \\
0 & 0 & 0
\end{array}\right] .
$$

This is the kinematic model on the Lie group for a unicycle-type robot. For one robot with a certain pose $\left(x_{I}, y_{I}, \theta_{I}\right)$, a control vector $(u, \omega)$ results in a unique control input matrix $\hat{\xi}$ to update the robot's motion.

\section{Formation CONTRol LAW ON $S E(2)$}

\subsection{Controller Design}

We consider $N$ unicycle-type mobile robots, and use $g_{i} \in S E(2)$ and $\bar{g}_{i} \in S E(2)(i=1, \cdots, N)$ to denote respectively the current configuration and the desired configuration of each robot. In fact, $g_{i}$ is the representation of the robot frame $\mathcal{F}_{R}$ shown in Figure 1 relative to the spatial frame $\mathcal{F}_{I}$. As introduced in the previous section, the evolution of system $g_{i}$ can be expressed by

$$
\dot{g}_{i}=\hat{\xi}_{i} g_{i}
$$

where $\hat{\xi}_{i} \in s e(2)$ is the control input matrix. Let $g_{i j}$ be the configuration of the robot- $j$ frame relative to the robot- $i$ frame, then we have

$$
g_{j}=g_{i} g_{i j}
$$

Thus $g_{i j}=g_{i}^{-1} g_{j}$. We can use $\bar{g}_{i j}$ to represent the desired configuration of the robot- $j$ frame in the robot$i$ frame. Then the robots achieve a desired formation if their configurations satisfy the following equation for any $k=1, \cdots, N, k \neq i$

$$
\lim _{t \rightarrow \infty} g_{k}^{-1} g_{i}=\bar{g}_{k i}, i=1, \cdots, N .
$$

$\bar{g}_{k i} \in S E(2)$ is defined according to the task requirements and is often used to identify the geometric configuration of the formation. We study the movement of $g_{i}$ relative to $g_{j}$, so here we can consider provisionally $g_{j}=\bar{g}_{j}$, then $\bar{g}_{i j}$ could be written as $\bar{g}_{i}^{-1} g_{j}$. Thus we have

$$
\begin{aligned}
\bar{g}_{i}^{-1} g_{j}=\bar{g}_{i}^{-1} \bar{g}_{j} & =\bar{g}_{i}^{-1}\left(\bar{g}_{k} \bar{g}_{k}^{-1}\right) \bar{g}_{j} \\
& =\left(\bar{g}_{k}^{-1} \bar{g}_{i}\right)^{-1}\left(\bar{g}_{k}^{-1} \bar{g}_{j}\right)=\left(\bar{g}_{k i}\right)^{-1} \bar{g}_{k j}
\end{aligned}
$$

which gives $\bar{g}_{i}=g_{j}\left(\bar{g}_{k j}\right)^{-1} \bar{g}_{k i}$. Then for robot- $i$ (in the local frame $g_{i}$ ), the needed transformation of robot- $i$ 
from the current configuration to the desired configuration while considering the current configuration of robot- $j$ is

$$
\tilde{g}_{i \_j}=g_{i}^{-1} g_{j}\left(\bar{g}_{k j}\right)^{-1} \bar{g}_{k i} .
$$

To simplify the notations, we note $\tilde{g}_{i j}$ instead of $\tilde{g}_{i \_j}$. In [1], noting $\tilde{x}_{i j}=\log \tilde{g}_{i j}$, a control law for agents, which have holonomic constraints, is proposed as

$$
\hat{\xi}_{i}=\frac{c}{a_{i}} \sum_{j=1}^{N} a_{i j} \tilde{x}_{i j}, i=1, \cdots, N
$$

where $a_{i j}$ is the element in the adjacency matrix $\mathcal{A}$ and $a_{i}=\sum_{j=1}^{N} a_{i j}$. However, in our MRS, nonholonomic constraints are associated with unicycle-type robots, so we develop a new nonlinear control approach. From the matrix $\tilde{g}_{i j}$, we could know the position error and orientation error $\tilde{x}_{i j}, \tilde{y}_{i j}, \tilde{\theta}_{i j}$. We suppose that the relative configuration of $\bar{g}_{i}$ with respect to the robot frame $g_{i}$ is denoted by $\bar{g}_{i i}$. Then $\bar{g}_{i i}$ could be obtained by the the mean function $M: \underbrace{S E(2) \times \cdots \times S E(2)}_{N-1} \rightarrow$

$S E(2),\left(\tilde{g}_{i 1}, \cdots, \tilde{g}_{i, i-1}, \tilde{g}_{i, i+1}, \cdots, \tilde{g}_{i N}\right) \mapsto \tilde{g}_{i i}$. This function means to get the weighted arithmetic mean of all the arguments, that is, if we note

$$
\tilde{g}_{i j}=\left[\begin{array}{ccc}
\cos \tilde{\theta}_{i j} & -\sin \tilde{\theta}_{i j} & \tilde{x}_{i j} \\
\sin \tilde{\theta}_{i j} & \cos \tilde{\theta}_{i j} & \tilde{y}_{i j} \\
0 & 0 & 1
\end{array}\right]_{j=1, \cdots, N, j \neq i},
$$

then $\tilde{x}_{i i}, \tilde{y}_{i i}, \tilde{\theta}_{i i}$ are given by:

$$
\Delta_{i i}=\frac{1}{a_{i}} \sum_{j=1}^{N} a_{i j} \Delta_{i j}, \quad j \neq i, \Delta=\tilde{x}, \tilde{y}, \tilde{\theta}
$$

where $a_{i j}$ is the element of adjacency matrix $\mathcal{A}$ and $a_{i}=\sum_{j=1}^{n} a_{i j}$. When $\tilde{x}_{i i}, \tilde{y}_{i i}$ and $\tilde{\theta}_{i i}$ are obtained, $\tilde{g}_{i i}$ can be rewritten as

$$
\tilde{g}_{i i}=\left[\begin{array}{ccc}
\cos \tilde{\theta}_{i i} & -\sin \tilde{\theta}_{i i} & \tilde{x}_{i i} \\
\sin \tilde{\theta}_{i i} & \cos \tilde{\theta}_{i i} & \tilde{y}_{i i} \\
0 & 0 & 1
\end{array}\right] .
$$

We take the inverse of the matrix $\tilde{g}_{i i}^{-1}$ which represents the relative configuration of $g_{i}$ with respect to the desired configuration $\bar{g}_{i}$ when the predefined communication topology is considered. Let us consider Figure 2, where the unknowns are annotated in the list of symbols after the article.

$O^{\prime} X^{\prime} Y^{\prime}$ is the frame of the desired configuration of robot $i$, and $(A, \theta)$, related to $\tilde{g}_{i i}^{-1}$, is the current pose of robot $i$ in the frame $O^{\prime} X^{\prime} Y^{\prime}$. In this frame, we assume a circle of radius $|r|$, denoted by $C_{B}$, and then we propose a control law to drive the robot to the origin with the help of this circle.

The absolute value $|r|$ is always positive, and it is supposed appropriately according to the initial conditions. $r$ is signed: when the robot is located in the lower half-plane, $r=-|r|$ and thus the angle $\alpha$ is also

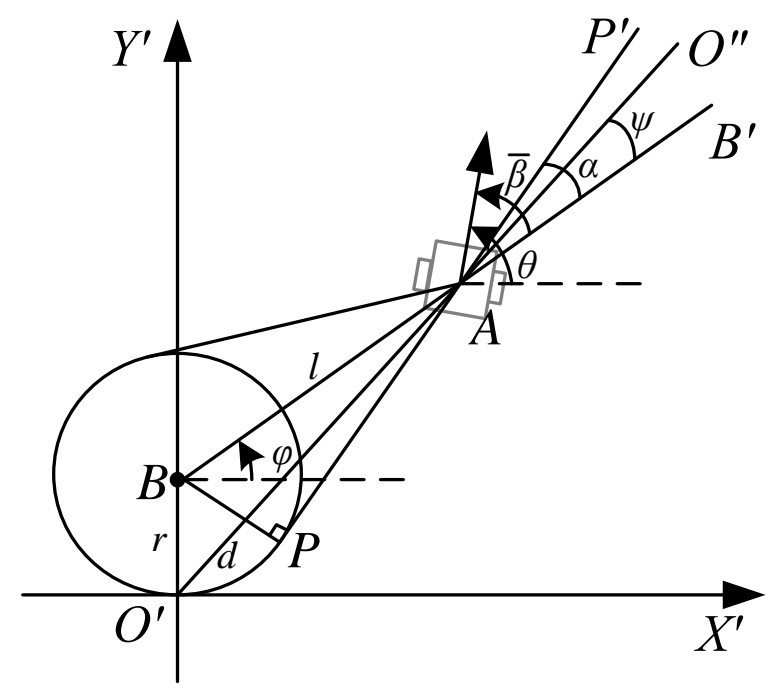

Figure 2. Geometrical relations between the robot actual configuration and the desired configuration.

negative. The coordinate $r$ is determined according to the following rules:

$$
\begin{cases}r \text { is chosen arbitrarily } & \\ \text { without changing sign } & \text { if }|r| \leq l / 2, \\ r=\frac{-y+\operatorname{sgn} y \sqrt{4 y^{2}+3 x^{2}}}{3} & \text { if }|r|>l / 2\end{cases}
$$

where the function "sgn" is defined as:

$$
\operatorname{sgn} x= \begin{cases}1, & x \geq 0 \\ -1, & x<0\end{cases}
$$

We denote $\beta=\arcsin \sin \bar{\beta}$, then the local control law is proposed as follows:

$$
\left\{\begin{array}{l}
u=-\operatorname{sgn} \cos \bar{\beta} \lambda l \\
\omega=\frac{u}{|r|}(\beta-\alpha)
\end{array}\right.
$$

where $\lambda$ is a positive constant. From the proposed law, we have $u_{i}$ and $\omega_{i}$, then the control input matrix of robot $i$ is obtained from Equation 6

\subsection{STABility ANALYSIS}

From the previous section, we know that $\tilde{g}_{i i}$ is the representation of $\bar{g}_{i}$ in frame $g_{i}$, while its inverse $\tilde{g}_{i i}^{-1}$ is the representation of $g_{i}$ in frame $\bar{g}_{i}$. To explain the convergence of $g_{i}$ to $\bar{g}_{i}$, we just need to prove that $\bar{g}_{i i}^{-1}$ converges to the origin, which is also the identity matrix $I$. To prove this, with the help of the notations depicted in Figure 2, we will divide the movement of each robot into three phases.

Phase 1. $l \geq 2|r|, \beta-\alpha \neq 0$.

Lemma 1. If we choose a convenient $r$ which satisfies $l \geq 2|r|$, then the angle between the direction of movement and one tangent of the circle $C_{B}$ converges to 0 , that is $\delta=|\beta-\alpha| \rightarrow 0$. 
Proof. If $r>0$, we have $\delta=|\beta-\alpha|=\sqrt{(\beta-\alpha)^{2}}$, then

$$
\dot{\delta}=\frac{\beta-\alpha}{\sqrt{(\beta-\alpha)^{2}}}(\dot{\beta}-\dot{\alpha})=\operatorname{sgn}(\beta-\alpha)(\dot{\beta}-\dot{\alpha}) .
$$

Because $\beta=\arcsin \sin \bar{\beta}=\arcsin \sin \theta-\varphi$, so

$$
\begin{aligned}
\dot{\beta}=\frac{1}{1-\sin ^{2}(\theta-\varphi)} \cos (\theta-\varphi) & (\dot{\theta}-\dot{\varphi}) \\
= & \operatorname{sgn} \cos \bar{\beta}(\dot{\theta}-\dot{\varphi}) .
\end{aligned}
$$

Consider the coordinate transformation into polar coordinates, we have $\dot{\varphi}=u \sin \beta / l$ and $\dot{l}=u \cos \beta$.

We distinguish three cases:

(1.) Case $\bar{\beta} \in\left[-\frac{\pi}{2}, \frac{\pi}{2}\right]$. In this case, the control law is $u=-\lambda l, \omega=-\lambda l(\beta-\alpha) / r$. And

$$
\begin{gathered}
\sin \alpha=\frac{r}{l} \\
\Rightarrow \dot{\alpha}=-\frac{r \dot{l}}{l^{2} \cos \alpha}=\frac{\lambda r \cos \beta}{l \cos \alpha}=\lambda \cos \beta \tan \alpha .
\end{gathered}
$$

Then we have

$$
\begin{array}{r}
\dot{\delta}=\operatorname{sgn}(\beta-\alpha)\left(\omega-\frac{u \sin \beta}{l}-\lambda \cos \beta \tan \alpha\right) \\
=\operatorname{sgn}(\beta-\alpha) \lambda\left[-\frac{l}{r}(\beta-\alpha)+\sin \beta\right. \\
-\cos \beta \tan \alpha] .
\end{array}
$$

Suppose $E_{\beta}=-\frac{l}{r}(\beta-\alpha)+\sin \beta-\cos \beta \tan \alpha$. Because $\sin \alpha=r / l$ and $l \geq 2 r$, so $\frac{d E_{\beta}}{d \beta}<0$. Then we can say that $E_{\beta}$ is a monotonically decreasing function about $\beta$, and $\beta=\alpha$ is the unique zero value point of $E_{\beta}$. Hence:

- if $-\frac{\pi}{2} \leq \beta<\alpha$, then $E_{\beta}>0, \beta-\alpha<0$ and $\dot{\delta} \leq 0$;

- if $\alpha \leq \beta \leq \frac{\pi}{2}$, then $E_{\beta} \leq 0, \beta-\alpha>0$ and $\dot{\delta} \leq 0$.

So $\delta$ converges monotonically to 0 .

(2.) Case $\bar{\beta} \in\left(\frac{\pi}{2}, \pi\right]$. We have $\beta=\pi-\bar{\beta} \in\left(0, \frac{\pi}{2}\right]$, and the control law is $u=\lambda l, \quad \omega=\frac{\lambda l}{r}(\pi-\bar{\beta}-\alpha)$.

In this case, we get

$$
\begin{array}{r}
\dot{\delta}=\operatorname{sgn}(\beta-\alpha) \lambda\left[\operatorname { s g n } \operatorname { c o s } \overline { \beta } \left(\frac{l}{r}(\pi-\bar{\beta}-\alpha)\right.\right. \\
-\sin \bar{\beta})+\cos \bar{\beta} \tan \alpha] .
\end{array}
$$

Suppose $E_{\beta}=\operatorname{sgn} \cos \bar{\beta}\left[\frac{l}{r}(\pi-\bar{\beta}-\alpha)-\sin \bar{\beta}\right]+$ $\cos \bar{\beta} \tan \alpha$, then

$$
\frac{d E_{\beta}}{d \beta}=-\frac{l}{r}+\cos \beta+\sin \beta \tan \alpha<0 .
$$

$\alpha=\beta=\pi-\bar{\beta}$ is the equilibrium point of $E_{\beta}$, so $\delta$ converge monotonically to 0 .

(3.) Case $\bar{\beta} \in\left[-\pi,-\frac{\pi}{2}\right)$. We have $\beta=-\pi-\bar{\beta}$, we can get a similar result to case 2 .

If $r<0$, the same calculus leads to the same results. Hence we have the conclusion $\delta=|\beta-\alpha| \rightarrow 0$.

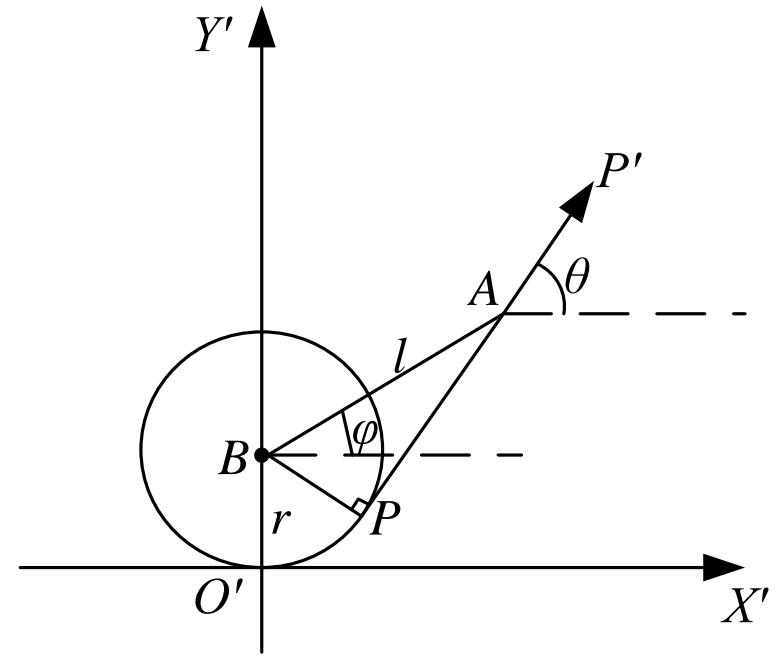

Figure 3. Movement in phase 3.

Phase 2. $l \geq 2|r|, \delta=\beta-\alpha=0$.

Because of the regulation of phase 1 , in this phase, the robot will moves towards the origin along the tangent of circle $C_{B}$, thus $\delta=0$ and $\omega=u(\beta-$ $\alpha) /|r|=0$.

Lemma 2. Suppose $d=\left|O^{\prime} A\right|$ and the Lyapunov function is chosen as $V=\frac{1}{2} d^{2}+\frac{1}{2} \theta^{2}$. If the robot moves towards the origin along the tangent of circle $C_{B}$, then $\dot{V}<0$.

Proof. Consider the polar coordinates, we have

$$
\dot{d}=u \cos (\bar{\beta}-\psi) \operatorname{sgn} x \operatorname{sgn} y=\cos (\bar{\beta}-\psi) s_{x} s_{y},
$$

where $s_{x}$ is $\operatorname{sgn} x$ for short. We find that if $u<0$, then $0<|\bar{\beta}| \leq \frac{\pi}{6}$; if $u>0$, then $\frac{5 \pi}{6} \leq|\bar{\beta}|<\pi$.

Angle $\psi$ is always positive. If $l=2|r|, \psi$ is maximal: $\psi_{\max }=\arccos (7 / 8) \approx 0.5054$, then no matter what sign $x$ and $y$ have, we have

$$
\dot{d}=-\operatorname{sgn} \cos \bar{\beta} \lambda l \cos \left(\bar{\beta}-\psi s_{x} s_{y}\right)<0 .
$$

In this phase, $\delta=0$, so $\dot{\theta}=\omega=0$. Hence $\dot{V}=$ $d \dot{d}+\theta \dot{\theta}<0$. The lemma is proved.

Phase 3. $l=2|r|$.

In this phase, we have always $l=2 r$ and $\beta=\alpha=$ $\frac{\pi}{6}$ (shown in Figure 3). We use $y(x)$ to represent the movement of the robot and suppose $r \geq 0$. The case $r<0$ could be studied in the same way and the same conclusion will be obtained. $(x, y)$ is the position of point $A$.

Theorem 1. Suppose that one robot, with the velocity defined by the proposed control law (Equation 13), moves towards the origin along the tangent of the circle $C_{B}$ (Figure 3) of which the radius $|r|$ satisfies $l=2|r|$ and $r$ is determined by rule (Equation 12), then both $d$ and $\theta$ asymptotically converge to 0 . 
Proof. We consider first the case where $r>0$. In this case, $l=2 r,(x, y)$ satisfies the equation $x^{2}+(y-r)^{2}=$ $4 r^{2}$. Then we get $r=\frac{-y+\sqrt{3 x^{2}+4 y^{2}}}{3}$ (the negative solution is omitted). Using $B P \perp P A$, we could have

$$
y^{\prime}=\frac{d y}{d x}=-\frac{3 x+\sqrt{3}\left(4 y-\sqrt{3 x^{2}+4 y^{2}}\right)}{-3 \sqrt{3} x+4 y-\sqrt{3 x^{2}+4 y^{2}}} .
$$

This is an homogeneous differential equation. We suppose $y=z x$ and differentiate it about $x$ :

$$
\begin{array}{r}
\frac{d y}{d x}=z+x \frac{d z}{d x}=\frac{3 x+\sqrt{3}\left(4 y-\sqrt{3 x^{2}+4 y^{2}}\right)}{3 \sqrt{3} x-4 y+\sqrt{3 x^{2}+4 y^{2}}} \\
=\frac{3+\sqrt{3}\left(4 z-\sqrt{3+4 z^{2}}\right)}{3 \sqrt{3}-4 z+\sqrt{3+4 z^{2}}} .
\end{array}
$$

Simplify this result and get

$$
\frac{d x}{x}=\frac{3 \sqrt{3}-4 z+\sqrt{3+4 z^{2}}}{3+\sqrt{z}+4 z^{2}-(\sqrt{3}+z) \sqrt{3+4 z^{2}}} d z .
$$

Integrate it and get

$$
\begin{array}{r}
\ln |x|=\int \frac{3 \sqrt{3}-4 z+\sqrt{3+4 z^{2}}}{3+\sqrt{z}+4 z^{2}-(\sqrt{3}+z) \sqrt{3+4 z^{2}}} d z \\
=\frac{\sqrt{3}}{z}\left(-\sqrt{1+\frac{4}{3} z^{2}}-1\right)-\frac{1}{2} \ln \left(z^{2}+1\right) \\
+\operatorname{arctanh} \frac{z}{\sqrt{3+4 z^{2}}}+\text { const }
\end{array}
$$

where "const" is a constant and its value is determined by the initial conditions.

When $x>0, x=l \cos \varphi$, so

$$
\begin{aligned}
\dot{x}=l & \cos \varphi-l \dot{\varphi} \sin \varphi=-\lambda l \cos \beta \\
& -l \lambda \sin \beta \sin \varphi=-\frac{\lambda l}{2}(\sqrt{3}+\sin \varphi)<0 .
\end{aligned}
$$

$l=0$ is the equilibrium point, so $x \rightarrow 0$. This is also the conclusion for $x<0$. So we just need to consider the right neighborhood of the origin, hence

$$
\begin{array}{r}
x=\exp \left(\frac{\sqrt{3}}{z}\left(-\sqrt{1+\frac{4}{3} z^{2}}-1\right)-\frac{1}{2} \ln \left(z^{2}+1\right)\right. \\
\left.+\operatorname{arctanh} \frac{z}{\sqrt{3+4 z^{2}}}+\text { const }\right) .
\end{array}
$$

Thus

$$
\frac{d x}{d z}=x \frac{\left(-z^{3}+\sqrt{3} z^{2}+\sqrt{3}\right) \sqrt{3+4 z^{2}}+4 z^{2}+3}{z^{2}\left(z^{2}+1\right) \sqrt{3+4 z^{2}}} .
$$

Solve the equation $\frac{d x}{d z}=0$, get $z_{0}=1+\sqrt{3}$. And when $z<z_{0}, \frac{d x}{d z}>0$; when $z>z_{0}, \frac{d x}{d z}<0$. So in the right neighborhood of the origin, $\frac{d x}{d z}<0$. Hence if $z \rightarrow 0$, then $x \rightarrow 0$, and we have the approximate relation between $x$ and $z$ :

$$
\ln x=-\frac{2 \sqrt{3}}{z}-\frac{z}{\sqrt{3}}-\frac{z^{2}}{2}+\mathcal{O}\left(z^{3}\right)
$$

Now $z$ is very close to 0 , so the terms of higher orders could be omitted and we get

$$
\ln x=-\frac{2 \sqrt{3}}{z}=-\frac{2 \sqrt{3} x}{y} .
$$

Thus $y=-\frac{2 \sqrt{3} x}{\ln x} \rightarrow 0$ when $x \rightarrow 0$.

The inclination also converges to 0 , because

$$
\begin{aligned}
\tan \theta=\frac{d y}{d x} \sim \frac{d}{d x}( & \left.-2 \sqrt{3} \frac{x}{\ln x}\right) \\
= & -2 \sqrt{3}\left(\frac{1}{\ln x}-\frac{1}{\ln ^{2} x}\right),
\end{aligned}
$$

when $x \rightarrow 0, \tan \theta \rightarrow 0$, then $\theta \rightarrow 0$. From the proposed control law, we know that $l=0$ is the equilibrium point, and here it is demonstrated that when $l \rightarrow 0$, the limit of $d$ and $\theta$ are both 0 .

In the polar coordinate frame, we have

$$
i=u \cos \bar{\beta}=-\operatorname{sgn} \cos \bar{\beta} \lambda l \cos \bar{\beta}=-\lambda l|\cos \bar{\beta}|,
$$

hence $l \rightarrow 0$. With the trigonometric relations, we could prove that $\dot{V}=d \dot{d}+\theta \dot{\theta}<0$, and the gain $\lambda$ does not affect the stability.

When $r<0$, the same reasoning can be made. This completes the proof.

Suppose $D$ to be a length that has the same order of the workspace of the system and satisfies $l \leq D$ for any $t \in \mathbb{R}$ and any robot. The two wheel velocities are

$$
\omega_{1}=\frac{2 u+\omega L}{2 r_{w}}, \omega_{2}=\frac{2 u-\omega L}{2 r_{w}},
$$

and satisfy $\left|\omega_{1}\right| \leq \omega_{\max },\left|\omega_{2}\right| \leq \omega_{\max }$, then we get the range of $\lambda$ :

$$
0<\lambda \leq \lambda_{\max }=\frac{r_{w} \omega_{\max }}{D+\frac{2 \pi}{3} \mu R_{\text {chassis }}}
$$

where $R_{\text {chassis }}=L / 2$ is the radius of the robot chassis, and $\mu$ is a convenient number that satisfies $l /|r| \leq \mu$ for all $t$.

\subsection{Stability of FORMATION CONTROL}

Because of the nonholonomic constraints, if there is a bidirectional path between any two unicycle-type robots which are equipped with this local control law, the system will not converge, so we propose a rooted directed acyclic graph as the communication topology of the multi-robot system and the theorem below.

Theorem 2. If the communication topology between $N$ unicycle-type robots is a rooted directed acyclic graph, then the system (Equation 7) will achieve the desired formation (Equation 9) under the local control law (Equation 11, 13). Especially, each robot, in phase 3 , converges to the desired formation asymptotically.

Proof. There is no directed circle, so the root node (robot) will not receive any information and will be static. Let $K_{m}$ denote the set of the nodes (robots) 


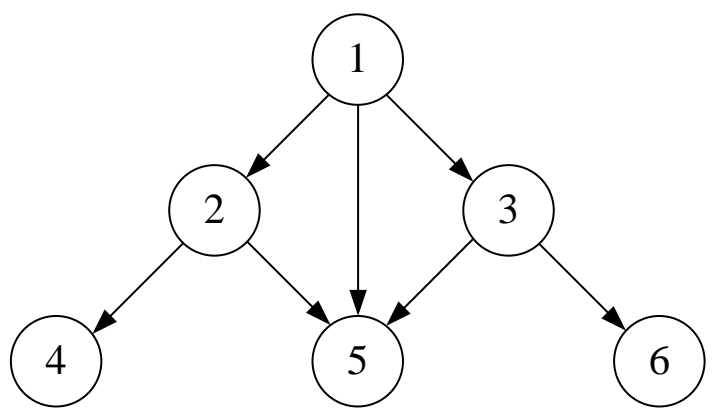

Figure 4. Communication topology of simulation.

to which there is a directed path from the root and this path consists of at most $m$ edges. Then $K_{0}$ has only one element - the root robot, denoted by $v_{0}$. The configuration of this robot in the fixed frame is denoted by $g_{0}=\bar{g}_{0}$. Then we use the mathematical induction method.

(1.) For $K_{1}$, suppose that there are $n_{1}$ elements in $K_{1}$. One element is denoted by $v_{1 i}$ where $1 \leq$ $i \leq n_{1}$, and the configuration of $v_{1 i}$ is denoted by $g_{1 i}$. Because $v_{1 i}$ receives information only from $v_{0}$, according to the lemmas above and theorem 1 we know that $\lim _{t \rightarrow \infty} g_{1 i}^{-1} g_{0}=\bar{g}_{1 i, 0}$.

(2.) For $K_{m}$, the elements in this set are denoted by $v_{m i}, 1 \leq i \leq c_{m}$ where $c_{m}$ is the cardinality of this set. $v_{m i}$ receives information from the nodes which are elements of $\bigcup K_{n, n \leq m-1}$ and have achieved the desired configurations. We use $j$ to denote the index numbers of these robots, that is, $v_{n_{j} j} \in K_{n_{j}} \subset$ $\bigcup K_{n, n \leq m-1}$. Then with the control law, $v_{m i}$ will converge to the desired configuration relative to $v_{n_{j} j}$, so

$$
\begin{aligned}
\lim _{t \rightarrow \infty} g_{m i}^{-1} g_{0} & =\lim _{t \rightarrow \infty} g_{m i}^{-1} g_{n_{j} j} g_{n_{j} j}^{-1} g_{0} \\
= & \left(\lim _{t \rightarrow \infty} g_{m i}^{-1} \bar{g}_{n_{j} j}\right)\left(\bar{g}_{n_{j} j}^{-1} \bar{g}_{0}\right) \\
& =\bar{g}_{\left(n_{j} j\right), m i}^{-1} \bar{g}_{\left(n_{j} j\right), 0}=\bar{g}_{m i, 0} .
\end{aligned}
$$

The topology graph is a finite graph, so all the robots will converge to the desired configuration relative to $v_{0}$. Then for any $v_{i}, v_{j}$, we have

$$
\lim _{t \rightarrow \infty} g_{i}^{-1} g_{j}=\lim _{t \rightarrow \infty} g_{i}^{-1} g_{0} g_{0}^{-1} g_{j}=\bar{g}_{0 i}^{-1} \bar{g}_{0 j}=\bar{g}_{i j} .
$$

Then the formation in Equation 9 is achieved.

\section{Simulation}

Let us consider a group of 6 unicycle-type robots which are located in a global frame, and we suppose that each robot could know its own position and orientation in the frame via GPS or via a camera which is installed above the work area. The initial pose of each is $p=$ $(x, y, \theta)$ where $(x, y)$ represents the robot position in

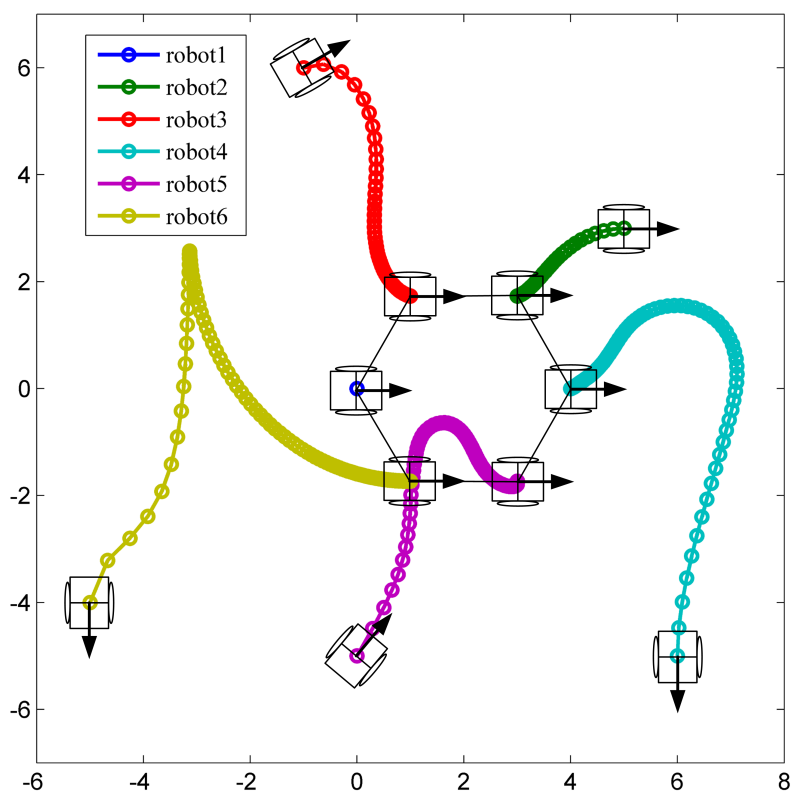

Figure 5. Trajectories of the 6 robots.

the global frame and angle $\theta$ indicates the orientation of the robot. The six initial poses are given by

$$
\begin{array}{ll}
p_{1}=(0,0,0), & p_{2}=(5,3,0), \\
p_{3}=(-1,6, \pi / 6), & p_{4}=(6,-5,-\pi / 2), \\
p_{5}=(0,-5, \pi / 3), & p_{3}=(-5,-4,-\pi / 2) .
\end{array}
$$

and the desired formation is a regular hexagon with side length of 2 . Let $c=1$, the sample time is $0.1 \mathrm{~s}$, and the maximum angular velocity of the wheels is $\omega_{\max }=5 \pi / \mathrm{s}$. The communication topology is given in Figure 4

Using Matlab, the results are obtained as shown in Figure 5 and 6. We observe that the six robots achieve the desired hexagonal formation: robot-1 has no information source, so it remains static. Other robots perform the trajectories according to the posture of their information source robots. Robots 4,5 and 6 achieve the desired configurations after robots 2 and 3 because of the communication topology shape. Figure 6 shows the evolution of the angles between the forward direction of each robot and the $\mathrm{X}$-axis of the global frame. We see that the six angles turn to the same value after some regulations of the configurations, which indicates the coordination of the robots' orientations. The rotation velocities become 0 at the end.

\section{Conclusions}

In this paper, we have studied the problem of formation control for a group of unicycle-type robots using a Lie group. A local control law based on $S E(2)$ for the robots is proposed, and the stability is analyzed. The problem is investigated under a rooted directed acyclic communication topology for a group of unicycle-type robots, and the behavior of the system is discussed. Some simulations of a 6 -robot system validate the 


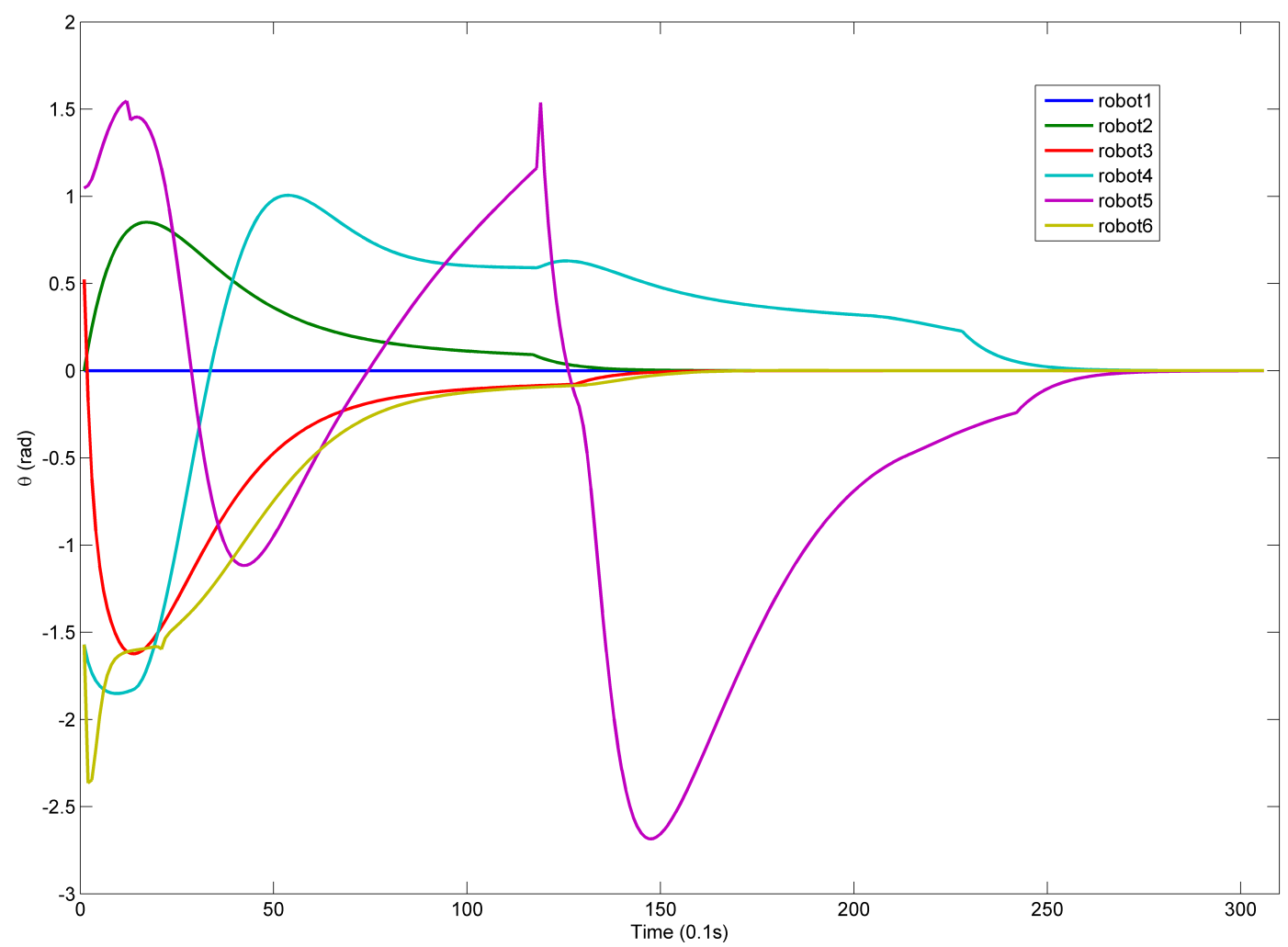

Figure 6 . Orientation of the 6 robots.

proposed control laws. The communication topology was supposed fixed. The case of switching topology, avoiding obstacles and an experiment on real robots will be studied in our future work.

\section{LIST OF SYMBOLS}

$u$ robot's longitudinal velocity $[\mathrm{m} / \mathrm{s}]$

$\omega$ robot's chassis instantaneous velocity of rotation $[\mathrm{rad} / \mathrm{s}]$

$\omega_{1}, \omega_{2}$ angular velocity of right and left wheel $[\mathrm{rad} / \mathrm{s}]$

$r_{\omega}$ radius of wheel $[\mathrm{m}]$

$L$ distance between the two actuated wheels [m]

$p=\left[x_{I}, y_{I}\right]^{T}$ position of robot in frame $\mathcal{F}_{I}$

$g_{i} \in S E(2)$ configuration of local frame attached to robot$i$ relative to frame $\mathcal{F}_{I}$

$g_{i j}$ configuration of robot- $j$ relative to the local frame attached to robot- $i$

$\bar{g}_{i j}$ desired configuration of robot- $j$ relative to robot- $i$

$B=(0, r)$ the centre of a circle of which the radius is $|r|$

$A=[x, y]^{T}$ position of robot $g_{i}$ in frame $O^{\prime} X^{\prime} Y^{\prime}$

$\theta$ orientation of robot $g_{i}$ relative to axis $O^{\prime} X^{\prime}$

$\varphi$ angle $\angle A B X^{\prime} \in[-\pi, \pi]$

$l$ distance between $A$ and $B$

$d$ distance between $A$ and $O^{\prime}$

$\bar{\beta}$ angle formed by $\overrightarrow{B A}$ and robot's orientation, $\in[-\pi, \pi]$

$\alpha \quad$ angle $\arcsin (r / l) \in[-\pi / 2, \pi / 2]$

$\psi$ angle $\angle B A O^{\prime} \in[0, \pi / 2]$

\section{ACKNOWLEDGEMENTS}

Youwei Dong was sponsored by the China Scholarship Council. Many thanks to members of the Math Stack Ex- change community, anonymous and otherwise, for helpful comments and suggestions.

\section{REFERENCES}

[1] R. Dong, Z. Geng. Consensus based formation control laws for systems on lie groups. in Systems \& Control Letters 62:104-111, 2013. DOI:10.1016/j.sysconle.2012.11.005

[2] A. Gautam, S. Mohan. A review of research in multi-robot systems. In Industrial and Information Systems(ICIIS), pp. 1-5. Chennai, Aug 2012. DOI:10.1109/ICIInfS.2012.6304778

[3] K.-K. Oh, H.-S. Ahn. Formation control of mobile agents based on distributed position estimation. in IEEE Transactions on Automatic Control 58(3):737742, March 2013. DOI:10.1109/TAC.2012.2209269

[4] H. Mehrjerdi, J. Ghommam, M. Saad. Nonlinear coordination control for a group of mobile robots using a virtual structure. in Mechatronics 21:1147-1155, 2011. DOI:10.1016/j.mechatronics.2011.06.006

[5] W. Ren. Consensus based formation control strategies for multi-vehicle systems. In Proceedings of the 2006 American Control Conference, pp. 4237-4242. Minnesota, June 2006. DOI:10.1109/ACC.2006.1657384.

[6] S. Mastellone, J. S. Mejía, D. M. Stipanović, M. W. Song. Formation control and coordinated tracking via asymptotic decoupling for lagrangian multi-agent systems. in Automatica 47:2355-2363, 2011. DOI:10.1016/j.automatica.2011.08.030

[7] R. Olfati-Saber, R. M. Murray. Distributed cooperative control of multiple vehicle formations using 
structural potential functions. In IFAC World Congress. Barcelona, 2002.

[8] K. D. Listmann, M. V. Masalawala, J. Adamy. Consensus for formation control of nonholonomic mobile robots, in robotics and automation. In Mathematical Physics, pp. 3886-3891. Kobe, May 2009. DOI:10.1109/ROBOT.2009.5152865

[9] P. Morin, C. Samson. Control of nonholonomic mobile robots based on the transverse function approach. in Transactions on Robotics 25(5):1058-1073, Oct 2009. DOI:10.1109/TRO.2009.2014123.

[10] R. M. Murray, Z. Li, S. S. Sastry. A Mathematical Introduction to Robotic Manipulation. CRC Press, 1994.

[11] F. W. Warner. Foundations of Differentiable Manifolds and Lie Groups. London: Scott, Foresman and Company, 1983.
[12] P. Coelho, U. Nunes. Lie algebra application to mobile robot control: a tutorial. in Robotica 21:483-493, 2003. DOI:10.1017/S0263574703005149.

[13] J. F. Cariñena, J. Clemente-Gallardo, A. Ramos. Motion on lie groups and its applications in control theory. in Mathematical Physics 51:159-70, Jul 2003. DOI:10.1016/S0034-4877(03)80010-1

[14] G. S. Chirikjian. Information theory on lie groups and mobile robotics applications. In IEEE International Conference on Robotics and Automation, pp. 2751-2757. Alaska, 2010. DOI:10.1109/ROBOT.2010.5509791.

[15] Z. Peng. Formation Control of Multi Nonhonomic Wheeled Mobile Robots. Ph.D. thesis, Ecole Centrale de Lille, june 2013.

[16] G. Wen. Distributed Cooperative Control for Multi-Agent Systems. Ph.D. thesis, Ecole Centrale de Lille, October 2012.

[17] B. Siciliano, O. Khatib. Handbook of Robotics. Springer, 2008. 